\title{
APPROXIMATION OF VISCOSITY SOLUTION BY MORPHOLOGICAL FILTERS
}

\author{
Denis PASQUignON ${ }^{1}$
}

\begin{abstract}
We consider in $\mathbb{R}^{2}$ all curvature equation $\frac{\partial u}{\partial t}=|D u| G(\operatorname{curv}(u))$ where $G$ is a nondecreasing function and $\operatorname{curv}(u)$ is the curvature of the level line passing by $\mathbf{x}$. These equations are invariant with respect to any contrast change $u \rightarrow g(u)$, with $g$ nondecreasing. Consider the contrast invariant operator $T_{t}: u_{o} \rightarrow u(t)$. A Matheron theorem asserts that all contrast invariant operator $T$ can be put in a form $(T u)(\mathbf{x})=\inf _{B \in \mathcal{B}} \sup _{\mathbf{y} \in B} u(\mathbf{x}+\mathbf{y})$. We show the asymptotic equivalence of both formulations. More precisely, we show that all curvature equations can be obtained as the iteration of Matheron operators $T_{h}^{n}$ where $h \rightarrow 0$ and $n \rightarrow \infty$ with $n h=t$.

Résumé. Nous considérons dans $\mathbb{R}^{2}$ les équations de courbure $\frac{\partial u}{\partial t}=|D u| G(\operatorname{curv}(u))$ où $G$ est une fonction croissante et $\operatorname{curv}(u)$ représente la courbure de la ligne de niveau passant par le point $\mathbf{x}$. Ces équations sont invariantes pour tout changement de contraste $u \rightarrow g(u)$, avec $g$ croissante. D'autre part, Matheron a prouvé que tout opérateur invariant par changement de contraste $T_{t}: u_{0} \rightarrow u(t)$ peut s'exprimer comme un schéma inf-sup $(T u)(\mathbf{x})=\inf _{B \in \mathcal{B}} \sup _{\mathbf{y} \in B} u(\mathbf{x}+\mathbf{y})$. Nous démontrons l'équivalence asymtotique de ces deux approches. Plus précisément, nous prouvons que la solution de viscosité de toute équation de courbure est la limite d'opérateurs de Matheron itérés $T_{h}^{n}$ lorsque $h \rightarrow 0$ et $n \rightarrow \infty$ avec $n h=t$.
\end{abstract}

AMS Subject Classification. 65N12, 35K65, 53C21, $76 \mathrm{~T} 05$.

Received July 7, 1998. Revised March 24, 1999.

\section{INTRODUCTION}

In image analysis, one of the most basic tasks is to smooth an image $u_{0}(\mathbf{x})$ for noise removal and shape simplification. Such a smoothing should preserve as much as possible the essential features of an image. This requirement is most easily formalized in terms of invariance. Two invariances requirements are basic in this context: given a smoothing operator $T$, it should commute with contrast changes, that is, increasing functions. Indeed, for physical and technological reasons, most digital images are known up to a contrast change. The second obvious requirement is geometric invariance: since the position of the camera is in general arbitrary or unknown, the operator $T$ should commute with translations, rotations, and, when possible, with affine and even projective transforms of the image plane.

There are some limitations, however, to the extent of invariance compatible with smoothing. A classical theorem of Matheron [12] asserts that if $T$ is a monotonous operator commuting with translations and contrast

\footnotetext{
Keywords and phrases: Viscosity solutions, inf-sup scheme, morphological filter.

1 CEREMADE, Université de Paris Dauphine, place de Lattre de Tassigny, 75775 Paris Cedex 16, France;

e-mail: pasquig@pi.ceremade.dauphine.fr
}

(c) EDP Sciences, SMAI 1999 
changes, there exists a family of structuring elements $\mathcal{B}$ such that

$$
(T u)(\mathbf{x})=\inf _{B \in \mathcal{B}} \sup _{\mathbf{y} \in B} u(\mathbf{x}+\mathbf{y}) .
$$

In the following, given a family $\mathcal{B}$, we note

$$
\begin{aligned}
& \left(I S_{h} u\right)(\mathbf{x})=\inf _{B \in h \mathcal{B}} \sup _{\mathbf{y} \in \mathbf{x}+B} u(\mathbf{y}), \\
& \left(S I_{h} u\right)(\mathbf{x})=\sup _{B \in h \mathcal{B}} \inf _{\mathbf{y} \in \mathbf{x}+B} u(\mathbf{y}), \\
& \left(A_{h} u\right)(\mathbf{x})=\left(I S_{h} \circ S I_{h} u\right)(\mathbf{x}) .
\end{aligned}
$$

On the other hand, several contrast invariant image operators based on PDE's have been proposed in recent years $[1,3,4]$, basically mean curvature motion $\left(\frac{\partial u}{\partial t}=|D u| \operatorname{curv}(u)\right)$ which commutes with all isometries and contrast changes and the so-called Affine Morphological Scale Space (AMSS), that is $\left(\frac{\partial u}{\partial t}=|D u|(\operatorname{curv}(u))^{\frac{1}{3}}\right)$. In [11], a multiscale analysis of shapes based on $\frac{\partial u}{\partial t}=|D u|(-\alpha+\operatorname{curv}(u)), \alpha>0$, was proposed for shape encoding. In [4], Catte et al. established a link between curvature equations and Matheron operators by proving that if we choose adequately $\mathcal{B}$, then suitably rescaled iterated Matheron filters converge to the viscosity solution of the mean curvature equation. This result was extended to the AMSS curvature equation by [8] in 2D and more recently by Cao [3] in any dimension. These partial results and propositions have opened a range of mathematical questions, one of which we attempt to solve here. The general questions in dimension 2 we address are:

1) do all adequately rescaled and iterated Matheron filters converge to a curvature equation?

2) Conversely, can all curvature equations $\frac{\partial u}{\partial t}=|D u| G(\operatorname{curv}(u))$ with $G$ a nondecreasing function, be obtained as the limit of iterated Matheron filters, as soon as they have a unique viscosity solution?

In [9], Ishii and Souganidis proved that such curvature equations with an arbitrary nondecreasing function $G$ have a viscosity solution theory yielding existence and uniqueness. Guichard and Morel give a partially positive answer to question 1). More precisely, they give a consistency result, which implies the convergence thanks to a theorem of Barles and Souganidis [2] about the convergence of approximation schemes.

In this paper, we answer positively question 2). More precisely, we prove the two following theorems.

Theorem 1.1. Given a function $G$ continuous, nondecreasing, there exists a family $\mathcal{B}_{\epsilon, M}, \epsilon, M>0$, such that the function defined with the alternate operator associated with $\mathcal{B}_{\epsilon, M}$

$$
u_{h, \epsilon, M}(\mathbf{x}, n h)=\left(A_{h}^{n} u\right)(\mathbf{x})
$$

tends uniformly on any compact set to the viscosity solution $u$ of

$$
\frac{\partial u}{\partial t}=|D u| G(\operatorname{curv}(u))
$$

when $\epsilon \rightarrow 0, M \rightarrow \infty$ and $n h \rightarrow t:$

$$
\lim _{\epsilon \rightarrow 0} \lim _{M \rightarrow \infty} \lim _{n h \rightarrow t} u_{h, \epsilon, M}(\mathbf{x}, n h)=u(t, x)
$$


Theorem 1.2. Let $\gamma \in[0 ;+\infty[$. There exists a family $\mathcal{B}$ such that the function

$$
u_{h}(\mathbf{x}, n h)=\left(A_{h}^{n} u\right)(\mathbf{x})
$$

tends uniformly when $n h \rightarrow t$ on any compact set to the viscosity solution $u(\mathbf{x}, t)$ of

$$
\frac{\partial u}{\partial t}=|D u|(\operatorname{curv}(u))^{\gamma} .
$$

The proof of Theorem 1.1 is easy thanks to a result of Ishii and Souganidis [9] but the family $\mathcal{B}_{\epsilon, M}$ depends on two parameters. Whereas with Theorem 1.2, the case of a power function $G(s)=\left|s^{\gamma-1}\right| s$ can be treated with families which do not depend on parameters. The case $\gamma<1$ is difficult because the family $\mathcal{B}_{h}$ we get is non local.

Our plan is as follows. In Section 2 we review several aspects and results of image processing theory relevant to questions 1) and 2). In Section 3 we formulate the problem, give the definitions and review the main results of [9]. We adapt the result of Barles and Souganidis to the new definition of viscosity solutions. In Section 4 we give a consistency theorem for Matheron filters. In Section 5, we approximate the viscosity solution in the general case. In Section 6, we construct a Matheron filter adapted to curvature equations with a power larger and smaller than 1 (both cases are quite different).

\section{IMAGE PROCESSING}

In 1983, Witkin and Koenderink [10,15] introduced the concept of scale space in image processing. It consists of a family of operators $\left(T_{t}\right)_{t \geq 0}$ which associates with an image modelled as a real valued function $u_{o}$ in $\mathbb{R}^{n}$ (in practice $n=2,3)$ a sequence of smoothed images $T_{t}\left(u_{o}\right)(\mathbf{x})=u(\mathbf{x}, t)$. We call $t$ the scale parameter. A complete axiomatization was presented by Alvarez et al. [1] who proved that if a scale space satisfies some stability and invariance properties, then $u(\mathbf{x}, t)$ is viscosity solution of

$$
\frac{\partial u}{\partial t}=|D u| G(\operatorname{curv}(u)) \text { and } u(\mathbf{x}, 0)=u_{o}(\mathbf{x}),
$$

where $G$ is any nondecreasing continuous function with respect to its argument and $\operatorname{curv}(u)$ is the curvature of the level line of the image $u(\mathbf{x}, t)$ passing by $\mathbf{x}$.

Among curvature equation used in image processing, let us mention:

- $G(s)=1$, we get an erosion, $G(s)=-1$, we get an dilation (in the Matheron sense).

- $G(s)=s$, we get the mean curvature motion.

- $G(s)=s^{\gamma}$ (that is $|s|^{\gamma-1} s$ ), then the associated scale space is scale invariant (commutes with zooms).

- $G(s)=s^{\frac{1}{3}}$, we get the AMSS [1] which is affine invariant.

- $G(s)=1+s$, we get a reaction diffusion equation introduced in image processing by Kimia et al. [11]. This kind of equation is used to compute the skeleton of a shape provided one adds a stopping test in order to prevent the shape from breaking [13].

\section{THE VISCOSITY SOLUTION FRAMEWORK}

In this section, we review and adapt several results in viscosity solution theory which we shall use in the following section. 

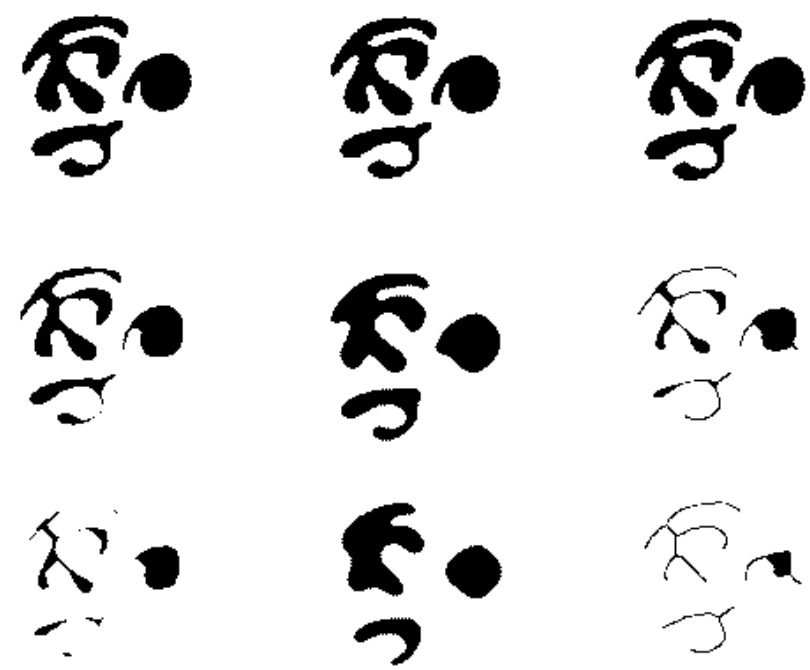

Figure 1. On the left column an erosion, on the middle mean curvature motion and on the right the skeletonization of the shapes.

We consider the nonlinear equation

$$
\frac{\partial u}{\partial t}=|D u| G(\operatorname{curv}(u)) \text { in } Q_{T}=\Omega \times(0, T)
$$

with $T>0 . \Omega$ is an open subset of $\mathbb{R}^{2}, u$ is the unknown funtion $u: \Omega \times(0, T) \rightarrow \mathbb{R}, G$ is a nondecreasing continuous function, $D u(\mathbf{x})$ is the spatial gradient and $\operatorname{curv}(u)$ is the curvature of the level line passing by $\mathbf{x}$,

$$
\operatorname{curv}(u)=\operatorname{div}\left(\frac{D u}{|D u|}\right)=\frac{u_{x x} u_{y}^{2}-2 u_{x} u_{y} u_{x y}+u_{y y} u_{x}^{2}}{\left(u_{x}^{2}+u_{y}^{2}\right)^{3 / 2}}
$$

Ishii and Souganidis [9] defined a new class of admissible test functions in the definition of viscosity solutions. To this end, let us denote by $\mathcal{F}(G)$ the set of functions $f \in C^{2}([0,+\infty))$ such that $f(0)=f^{\prime}(0)=f^{\prime \prime}(0)=0$ and $f^{\prime \prime}(r)>0$ for $r>0$ and such that

$$
\lim _{p \rightarrow 0} f^{\prime}(|p|) G\left(\frac{1}{|p|}\right)=\lim _{p \rightarrow 0} f^{\prime}(|p|) G\left(\frac{-1}{|p|}\right)=0 .
$$

Ishii and Souganidis [9] prove that this set is a nonempty cone.

Definition 3.1. Let $\mathcal{O}$ be an open subset of $Q_{T}$. A function $\phi \in C^{2}(\mathcal{O})$ is admissible for $G$ if for any $\left(\mathbf{x}_{o}, t_{o}\right) \in \mathcal{O}$ such that $D \phi\left(\mathbf{x}_{o}, t_{o}\right)=0$, there is a constant $\delta>0$ and functions $f \in \mathcal{F}(G)$ and $\omega \in C((0,+\infty))$ satisfying $\lim _{r \rightarrow 0} \frac{\omega(r)}{r}=0$, such that, for all $(\mathbf{x}, t) \in B\left(\left(\mathbf{x}_{o}, t_{o}\right), \delta\right)$

$$
\left|\phi(\mathbf{x}, t)-\phi\left(\mathbf{x}_{o}, t_{o}\right)-\phi_{t}\left(\mathbf{x}_{o}, t_{o}\right)\left(t-t_{o}\right)\right| \leq f\left(\left|\mathbf{x}-\mathbf{x}_{o}\right|\right)+\omega\left(\left|t-t_{o}\right|\right) .
$$

We note $\mathcal{A}(G)$ the set of admissible functions.

We recall the definition of the upper semicontinuous envelope $u^{*}$ and the lower semicontinuous envelope $u_{*}$ of a function $u$

$$
u^{*}(\mathbf{x}, t)=\limsup _{(\mathbf{y}, s) \rightarrow(\mathbf{x}, t)}(u(\mathbf{y}, s)) \text { and } u_{*}(\mathbf{x}, t)=\liminf _{(\mathbf{y}, s) \rightarrow(\mathbf{x}, t)}(u(\mathbf{y}, s)) .
$$


Definition 3.2. (Ishii-Souganidis) A function $u(\mathbf{x}, t): \mathcal{O} \rightarrow \mathbb{R} \cup\{-\infty\}$ is a viscosity subsolution of (1) in $\mathcal{O}$ if $u^{*}<+\infty$ in $\mathcal{O}$ and for all $\phi \in \mathcal{A}(G)$ and all local finite maximum points $\left(\mathbf{x}_{o}, t_{o}\right)$ of $u^{*}-\phi$,

$$
\begin{cases}\frac{\partial \phi}{\partial t}\left(\mathbf{x}_{o}, t_{o}\right)-\left|D \phi\left(\mathbf{x}_{o}, t_{o}\right)\right| G\left(\operatorname{curv}(\phi)\left(\mathbf{x}_{o}, t_{o}\right)\right) \leq 0 & \text { if } D \phi\left(\mathbf{x}_{o}, t_{o}\right) \neq 0 \\ \frac{\partial \phi}{\partial t}\left(\mathbf{x}_{o}, t_{o}\right) \leq 0 & \text { if } D \phi\left(\mathbf{x}_{o}, t_{o}\right)=0 .\end{cases}
$$

A function $u(\mathbf{x}, t): \mathcal{O} \rightarrow \mathbb{R} \cup\{-\infty\}$ is a viscosity supersolution of (1) in $\mathcal{O}$ if $u_{*}>-\infty$ in $\mathcal{O}$ and for all $\phi \in \mathcal{A}(G)$ and all local finite maximum points $\left(\mathbf{x}_{o}, t_{o}\right)$ of $u_{*}-\phi$,

$$
\begin{cases}\frac{\partial \phi}{\partial t}\left(\mathbf{x}_{o}, t_{o}\right)-\left|D \phi\left(\mathbf{x}_{o}, t_{o}\right)\right| G\left(\operatorname{curv}(\phi)\left(\mathbf{x}_{o}, t_{o}\right)\right) \geq 0 & \text { if } D \phi\left(\mathbf{x}_{o}, t_{o}\right) \neq 0 \\ \frac{\partial \phi}{\partial t}\left(\mathbf{x}_{o}, t_{o}\right) \geq 0 & \text { if } D \phi\left(\mathbf{x}_{o}, t_{o}\right)=0 .\end{cases}
$$

A function $u(\mathbf{x}, t)$ is a viscosity solution of $(1)$ in $\mathcal{O}$ if $\mathrm{u}$ is both a viscosity subsolution and supersolution in $\mathcal{O}$.

We denote by $B U C\left(\mathbb{R}^{2}\right)$ the set of bounded uniformly continuous functions on $\mathbb{R}^{2}$.

Theorem 3.3. (Ishii-Souganidis) Consider the initial value problem with $G$ a non decreasing continuous function:

$$
\left\{\begin{array}{c}
\frac{\partial u}{\partial t}-|D u| G(\operatorname{curv}(u))=0 \\
u(\mathbf{x}, 0)=u_{o}(\mathbf{x})
\end{array}\right.
$$

Assume that $u_{o} \in B U C\left(\mathbb{R}^{2}\right)$. Then there is an unique viscosity solution $u \in B U C\left(\mathbb{R}^{2} \times(0, T)\right)$.

In the following, we need the result of Ishii and Souganidis [9] (Pr. 1.3).

Theorem 3.4. Let $G$ be a nondecreasing continuous function. Let us assume that $G_{n}$ converge locally uniformly to $G$, that $\mathcal{F}(G) \subset \mathcal{F}\left(G_{n}\right)$ for any $n$ and that for any $f \in \mathcal{F}(G)$,

$$
\liminf _{p \rightarrow 0, n \rightarrow \infty} f^{\prime}(|p|) G_{n}\left(\frac{1}{p}\right) \geq 0
$$

(resp.

$$
\left.\limsup _{p \rightarrow 0, n \rightarrow \infty} f^{\prime}(|p|) G_{n}\left(\frac{-1}{p}\right) \leq 0\right) .
$$

Let $u_{n}$ be a subsolution (resp. a supersolution) of

$$
\frac{\partial u_{n}}{\partial t}=\left|D u_{n}\right| G_{n}\left(\operatorname{curv}\left(u_{n}\right)\right) \text { in } \mathcal{O},
$$

and define $u^{*}$ and $u_{*}: \mathcal{O} \rightarrow \mathbb{R} \cup\{-\infty, \infty\}$ by

$$
\begin{aligned}
& u^{*}(z)=\limsup _{r \rightarrow 0}\left\{u_{n}(y),|y-z| \leq r, n>\frac{1}{r}\right\} \\
& u_{*}(z)=\liminf _{r \rightarrow 0}\left\{u_{n}(y),|y-z| \leq r, n>\frac{1}{r}\right\} .
\end{aligned}
$$


Assume that $u^{*}(z)<\infty$ (resp. $\left.u_{*}(z)>-\infty\right)$ for all $z \in \mathcal{O}$. Then $u^{*}$ (resp. $u_{*}$ ) is a subsolution (resp. a supersolution) of

$$
\frac{\partial u}{\partial t}=|D u| G(\operatorname{curv}(u)) \text { in } \mathcal{O} \text {. }
$$

Our purpose is to approximate the solution $u$ of Theorem 3.3 by an iterated scheme $T_{h}$ where $h$ is a scale parameter. To prove the convergence, we follow the argumentation developed by Barles and Souganidis [2]. Let $u_{o}$ be a bounded function on $\mathbb{R}^{2}$. We set

$$
u_{h}(\mathbf{x},(n+1) h)=T_{h} u_{h}(\mathbf{x}, n h)=T_{h}^{n+1} u_{o}(\mathbf{x}), u_{h}(\mathbf{x}, 0)=u_{o}(\mathbf{x})
$$

Definition 3.5. We note $B(\bar{\Omega})$ the set of bounded functions.

- Consistency

A scheme $T_{h}, h>0$ is consistent with

$$
\frac{\partial u}{\partial t}=|D u| G(\operatorname{curv}(u))
$$

if for any $u \in C^{\infty}(\bar{\Omega})$ and for any $\mathbf{x} \in \bar{\Omega}$ we have

$$
\frac{\left(T_{h} u\right)(\mathbf{x})-u(\mathbf{x})}{h}=|D u| G(\operatorname{curv}(u))+o_{\mathbf{x}}(1) \text { if } D u(\mathbf{x}) \neq 0 .
$$

If the convergence of $o_{\mathbf{x}}(1)$ is uniform on any compact set on which $D u(\mathbf{x}) \neq 0$, the scheme $T_{h}$ is said to be uniformly consistent with the PDE.

\section{- Monotonicity}

A scheme $T_{h}, h>0$ is locally monotone if there exists $r>0$ such that for any functions $u, v \in B(\bar{\Omega})$ with $u(\mathbf{y})>v(\mathbf{y})$ on $D(\mathbf{x}, r) \backslash\{\mathbf{x}\}$, one has

$$
T_{h} u(\mathbf{x}) \geq T_{h} v(\mathbf{x})+o(h) .
$$

\section{- Stability}

A scheme $T_{h}, h>0$ is stable if for any $u \in B(\bar{\Omega})$ and for any $h>0$ and $n, T_{h}^{n} u \in B(\bar{\Omega})$.

Theorem 3.6. (Barles-Souganidis) We consider a scheme $T_{h}, h>0$, uniformly consistent with $\frac{\partial u}{\partial t}=$ $|D u| G(\operatorname{curv}(u))$, monotone, stable and commuting with the addition of constants (i.e. $\left.T_{h}(u+C)=T_{h} u+C\right)$.

For any $\mathbf{x}_{o}$, we set

$$
u(\mathbf{x})=f\left(\left|\mathbf{x}-\mathbf{x}_{o}\right|\right) .
$$

Assume that for any $f \in \mathcal{F}(G)$,

$$
\lim _{h \rightarrow 0} \frac{T_{h} u\left(\mathbf{x}_{o}\right)}{h}=0 .
$$

Then when $n h \rightarrow t, u_{h}(\mathbf{x}, n h)$ tends uniformly on any compact set to the viscosity solution $u(\mathbf{x}, t)$ of

$$
\frac{\partial u}{\partial t}=|D u| G(\operatorname{curv}(u))
$$

The proof is an easy adaptation of Barles and Souganidis [2]. For a sake of completeness, we include this adaptation in the Appendix. 


\section{Consistency theorem for Matheron filters}

Theorem 4.1. Asymptotic behaviour. Let $\mathcal{B}$ be a family of structuring elements in $\mathbb{R}^{2}$ which is bounded and isotropic. Assume that each element $B \in \mathcal{B}$ is symmetric with respect to $O$.

We define the operators

$$
(T u)(\mathbf{x})=\sup _{B \in \mathcal{B}} \inf _{\mathbf{y} \in \mathbf{x}+B} u(\mathbf{y})
$$

and

We set

$$
\left(T_{h} u\right)(\mathbf{x})=\sup _{B \in h \mathcal{B}} \inf _{\mathbf{y} \in \mathbf{x}+B} u(\mathbf{y}) .
$$

with $u(x, y)=x+b y^{2}$.

$$
G(b)=T(u)(0)
$$

Assume that there exists $\gamma \geq 1$

$$
G(b) \simeq b^{\gamma} \text { when } b \rightarrow 0 .
$$

If $u$ is a $C^{n}$ function on $\mathbb{R}^{2}$ with $n \geq \gamma+2$,

- if $D u\left(\mathbf{x}_{o}\right) \neq 0$, then

$$
T_{h} u\left(\mathbf{x}_{o}\right)=u\left(\mathbf{x}_{o}\right)+h^{\gamma+1}\left|D u\left(\mathbf{x}_{o}\right)\right|\left(\frac{1}{2} \operatorname{curv} u\right)^{\gamma}+\mathcal{O}\left(h^{\gamma+2}\right) .
$$

The consistency is uniform on a compact set on which $D u(\mathbf{x}) \neq 0$.

- if $D u\left(\mathbf{x}_{o}\right)=0$, then

$$
T_{h} u\left(\mathbf{x}_{o}\right)=u\left(\mathbf{x}_{o}\right)+h^{2} \inf _{\mathcal{B}} \sup _{(x, y) \in B}\left(\lambda_{1} x^{2}+\lambda_{2} y^{2}\right)+\mathcal{O}\left(h^{3}\right) .
$$

$\lambda_{1}$ and $\lambda_{2}$ are the eigenvalues of $D^{2} u(\mathbf{x})$.

Proof. We only prove the case $D u\left(\mathbf{x}_{o}\right) \neq 0$, the other one is straightforward, see Guichard and Morel [8]. As $T\left(u-u\left(\mathbf{x}_{o}\right)\right)=T u-u\left(\mathbf{x}_{o}\right)$, we can choose $u\left(x_{o}\right)=0$. Moreover as $T$ is invariant by any isometry, it is possible to choose the origin $\mathrm{O}$ at $x_{o}$, and the orthogonal axes such that $\vec{i}=\frac{D u}{|D u|}$ if $D u\left(\mathbf{x}_{o}\right) \neq 0$. Let $\vec{j}$ be such that $(\vec{i}, \vec{j})$ is the orthonormal basis. Assume that $u$ is $C^{\gamma+2}$. In what follows, we note $(x, y)$ the coordinates of $\mathbf{x}$ in $(O, \vec{i}, \vec{j})$. Then, with these conventions,

$$
u(\mathbf{x})=p x+a x^{2}+b y^{2}+c x y+\sum_{2<k+l \leq \gamma+1} a_{k, l} x^{k} y^{l}+\mathcal{O}\left(|\mathbf{x}|^{\gamma+2}\right)
$$

with $p=|D u|(0)$, and $p>0$. Moreover we have

$$
\begin{aligned}
a & =\frac{1}{2} D^{2} u(\vec{i}, \vec{i}), \\
b & =\frac{1}{2} D^{2} u(\vec{j}, \vec{j})=\frac{1}{2}|D u| \operatorname{curv} u(0),
\end{aligned}
$$

and

We set

$$
c=D^{2} u(\vec{i}, \vec{j})
$$

$$
Q(x, y)=p x+a x^{2}+b y^{2}+c x y+\sum_{2<k+l \leq \gamma+1} a_{k, l} x^{k} y^{l}
$$


So we have

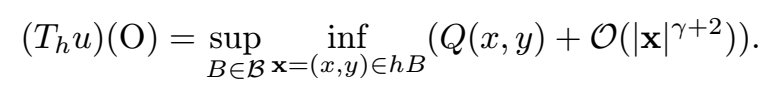

As $\mathcal{B}$ is bounded, there exists a constant $\mathrm{M}$ such that every element $\mathrm{B}$ of $\mathcal{B}$ is included in $D(O, M)$. Therefore if $\mathbf{x} \in h B$ then $|\mathbf{x}|<h M$.

As $T_{h}$ is monotone and commutes with addition of constant, we get

$$
T_{h} u(\mathrm{O})=T_{h}(Q(x, y))+\mathcal{O}\left(|h|^{\gamma+2}\right) .
$$

We have the following inequalities for the points $(x, y)$ of $h B$

$$
\begin{gathered}
x\left(p-\epsilon(x)\left[(|a|+|c|) M h+\sum_{2<k+l \leq \gamma+1,0<k}\left|a_{k, l}\right| M^{k+l-1} h^{k-1} h^{l}\right)\right]+y^{2}\left(b-\sum_{2<l<\gamma+1}\left|a_{0, l}\right|(M h)^{l-2}\right) \leq \\
x\left(p+\epsilon(x)\left[(|a|+|c|) M h+\sum_{2<k+l \leq \gamma+1,0<k}\left|a_{k, l}\right| M^{k+l-1} h^{k-1} h^{l}\right)\right]+y^{2}\left(b+\sum_{2<l<\gamma+1}\left|a_{0, l}\right|(M h)^{l-2}\right),
\end{gathered}
$$

and $\epsilon(x)$ is the sign of $x$ : if $x \leq 0, \epsilon(x)=-1$, and if $x>0, \epsilon(x)=1$.

Since the coefficients $a, c$ and $a_{k, l}$ are bounded and continuous on $D(O, M)$, we note $K$ and $K^{\prime}$ such that

$$
K=\sup _{D(O, M)}\left((|a|+|c|) M+\sum_{2<k+l \leq \gamma+1,0<l}\left|a_{k, l} M^{k+l-1}\right|\right),
$$

and

So we have

$$
K^{\prime}=\sup _{D(O, M)}\left(\sum_{2<l<\gamma+1}\left|a_{0, l}\right| M^{l-3}\right)
$$

Thus, for any $B \in \mathcal{B}$,

$$
\inf _{(x, y) \in h B}\left(p x-|x| K h+y^{2}\left(b-K^{\prime} h\right)\right) \leq \inf _{(x, y) \in h B} Q(x, y) \leq \inf _{(x, y) \in h B}\left(p x+|x| K h+y^{2}\left(b+K^{\prime} h\right)\right) .
$$

Since $B$ is symmetric with respect to $\mathrm{O}$, we deduce that it contains points $\mathbf{x}=(x, y)$ such that $x$ is negative. As $p>0$, we deduce that the infimum is reached for a negative $x$ for a small $h$. Therefore, we get

$$
\inf _{(x, y) \in h B}\left(p x-|x| K h+y^{2}\left(b-K^{\prime} h\right)\right)=\inf _{(x, y) \in h B \cap\{(x, y), x<0\}}\left(p x+x K h+y^{2}\left(b-K^{\prime} h\right)\right) .
$$

With the same argument,

$$
\inf _{(x, y) \in h B \cap\{(x, y), x<0\}}\left(p x+x K h+y^{2}\left(b-K^{\prime} h\right)\right)=\inf _{(x, y) \in h B}\left(p x+x K h+y^{2}\left(b-K^{\prime} h\right)\right) .
$$

Then,

$$
\inf _{(x, y) \in h B}\left(p x+x K h+y^{2}\left(b-K^{\prime} h\right)\right) \leq \inf _{(x, y) \in h B} Q(x, y) \leq \inf _{(x, y) \in h B}\left(p x-x K h+y^{2}\left(b+K^{\prime} h\right)\right) .
$$


So we deduce

$$
S I_{h}\left(x(p+K h)+y^{2}\left(b-K^{\prime} h\right)\right) \leq S I_{h}(Q(x, y)) \leq S I_{h}\left(x(p-K h)+y^{2}\left(b+K^{\prime} h\right)\right) .
$$

As $T_{h}\left(x+b y^{2}\right)(O)=h G(h b)$, we have

$$
S I_{h}\left(x(p-K h)+y^{2}\left(b+K^{\prime} h\right)\right)=h(p-K h) G\left(h \frac{b+K^{\prime} h}{p-K h}\right),
$$

we deduce that

and

$$
S I_{h}\left(x(p-K h)+y^{2}\left(b+K^{\prime} h\right)\right)=h^{\gamma+1} p\left(\frac{b}{p}\right)^{\gamma}+C_{1}\left(b, p, K, K^{\prime}\right) h^{\gamma+2},
$$

$$
S I_{h}\left(x(p+K h)+y^{2}\left(b-K^{\prime} h\right)\right)=h^{\gamma+1} p\left(\frac{b}{p}\right)^{\gamma}+C_{2}\left(b, p, K, K^{\prime}\right) h^{\gamma+2},
$$

$C_{1}$ and $C_{2}$ are continuous functions with respect to all their arguments. Then

$$
S I_{h}(Q(x, y))=h^{\gamma+1} p\left(\frac{b}{p}\right)^{\gamma}+\mathcal{O}\left(h^{\gamma+2}\right),
$$

thus

$$
S I_{h} u=h^{\gamma+1} p\left(\frac{b}{p}\right)^{\gamma}+\mathcal{O}\left(h^{\gamma+2}\right) .
$$

From this result, we deduce the consistency if $D u\left(\mathbf{x}_{o}\right) \neq 0$ :

$$
T_{h} u\left(\mathbf{x}_{o}\right)=u\left(\mathbf{x}_{o}\right)+h^{\gamma+1}\left|D u\left(\mathbf{x}_{o}\right)\right| G\left(\frac{1}{2} \operatorname{curv} u\right)+\mathcal{O}\left(h^{\gamma+2}\right) .
$$

In addition, consider $\Omega$ a neighborhood of $\mathbf{x}_{o}$ such that for any $\mathbf{x} \in \Omega|D u(\mathbf{x})| \neq 0$. We have for any $\mathbf{x} \in \Omega$

$$
h^{\gamma+1} p\left(\frac{b}{p}\right)^{\gamma}+C_{1}\left(b, p, K, K^{\prime}\right) h^{\gamma+2} \leq T_{h}(u)(\mathbf{x})-u\left(x_{o}\right) \leq h^{\gamma+1} p\left(\frac{b}{p}\right)^{\gamma}+C_{2}\left(b, p, K, K^{\prime}\right) h^{\gamma+2},
$$

and $b, p, K, K^{\prime}$ are continuous functions of $\mathbf{x}$, therefore $C_{1}$ and $C_{2}$ are bounded functions on $\Omega$. So we deduce that the consistency of $T_{h}$ is uniform in $\Omega$.

This theorem enables us to build in Section 6 a family $\mathcal{B}$ with which we define an inf-sup scheme converging to the viscosity solution when $G$ is a power function. But in the general case, we get the following equation if $D u\left(\mathbf{x}_{o}\right) \neq 0$ :

$$
T_{h} u\left(\mathbf{x}_{o}\right)=u\left(\mathbf{x}_{o}\right)+h\left|D u\left(\mathbf{x}_{o}\right)\right| G\left(\frac{1}{2} h \operatorname{curv} u\right)+o(h) .
$$

Since the scale parameter $h$ is linked to the curvature term $\operatorname{curv}(u), G\left(\frac{1}{2} h \operatorname{curv} u\right)$, it is impossible to separate these two terms when $G$ is not a power function. Instead of considering $\mathcal{B}_{h}$ as $h \mathcal{B}$, we give an other definition for the scaling of a family $\mathcal{B}_{h}$.

Definition 4.2. Let $\mathcal{B}$ be a family of structuring elements. $B \in \mathcal{B}_{h}$ if and only if there exists $B^{\prime} \in \mathcal{B}$ and $\theta \in[0,2 \pi]$ such that

where

$$
B=P_{\theta} A_{h} B^{\prime}
$$

$$
P_{\theta}=\left(\begin{array}{cc}
\cos (\theta) & -\sin (\theta) \\
\sin (\theta) & \cos (\theta)
\end{array}\right)
$$


and

$$
A_{h}=\left(\begin{array}{cc}
h & 0 \\
0 & h^{\frac{1}{2}}
\end{array}\right)
$$

Theorem 4.3. (Asymptotic behaviour 2). Let $\mathcal{B}$ a bounded and isotropic family of structuring elements of $\mathbb{R}^{2}$. Assume that each element $B \in \mathcal{B}$ is symmetric with respect to $O$.

Let $(T u)(\mathbf{x})=\inf _{B \in \mathcal{B}} \sup _{\mathbf{y} \in \mathbf{x}+B} u(\mathbf{y}),\left(\right.$ resp. $\left.(T u)(\mathbf{x})=\sup _{B \in \mathcal{B}} \inf _{\mathbf{y} \in \mathbf{x}+B} u(\mathbf{y})\right)$. Define the scheme

$$
\left(T_{h} u\right)(\mathbf{x})=\inf _{B \in \mathcal{B}_{h}} \sup _{\mathbf{y} \in \mathbf{x}+B} u(\mathbf{y})
$$

$\left(\right.$ resp. $\left.\left(T_{h} u\right)(\mathbf{x})=\sup _{B \in \mathcal{B}_{h}} \inf _{\mathbf{y} \in \mathbf{x}+B} u(\mathbf{y})\right)$, and set

$$
G(b)=T\left(x+b y^{2}\right)(0)
$$

Then if $u \in C^{\infty}\left(\mathbb{R}^{2}\right)$, if $D u\left(\mathbf{x}_{o}\right) \neq 0$, we have

$$
T_{h} u(\mathbf{x})=u\left(\mathbf{x}_{o}\right)+h\left|D u\left(\mathbf{x}_{o}\right)\right| G\left(\frac{1}{2} \operatorname{curv}(u)\left(\mathbf{x}_{o}\right)\right)+\mathcal{O}\left(h^{\frac{3}{2}}\right) .
$$

The consistency is uniform on a compact set on which $D u(\mathbf{x}) \neq 0$.

Proof. As $T\left(u-u\left(\mathbf{x}_{o}\right)\right)=T u-u\left(\mathbf{x}_{o}\right)$, we can choose $u\left(x_{o}\right)=0$. Moreover as $T$ is invariant by any isometry, it is possible to take the origin $\mathrm{O}$ at $\mathbf{x}_{o}$, and the orthogonal axes such that $\vec{i}=\frac{D u}{\mid D u}$ if $D u\left(\mathbf{x}_{o}\right) \neq 0$. Let $\vec{j}$ be such that $(\vec{i}, \vec{j})$ is an orthonormal basis. So we have with $\mathbf{x}=(x, y)$

$$
u(\mathbf{x})=p x+a x^{2}+b y^{2}+c x y+\mathcal{O}\left(|\mathbf{x}|^{3}\right)
$$

with $p=|D u|(0)>0$, and

$$
\begin{aligned}
a & =\frac{1}{2} D^{2} u(\vec{i}, \vec{i}), \\
b & =\frac{1}{2} D^{2} u(\vec{j}, \vec{j})=\frac{1}{2}|D u| \operatorname{curv} u(0),
\end{aligned}
$$

and

$$
c=D^{2} u(\vec{i}, \vec{j})
$$

We set

$$
Q(x, y)=p x+a x^{2}+b y^{2}+c x y
$$

We have for $B^{\prime} \in \mathcal{B}_{h}$

$$
\begin{gathered}
\left(x^{\prime}, y^{\prime}\right) \in B^{\prime} \Leftrightarrow \exists B \in \mathcal{B} \exists(x, y) \in B \exists \theta \in[0,2 \pi] \text { such that } \\
\left(\begin{array}{c}
x^{\prime} \\
y^{\prime}
\end{array}\right)=P_{\theta} A_{h}\left(\begin{array}{l}
x \\
y
\end{array}\right)
\end{gathered}
$$

or

$$
\left\{\begin{array}{l}
x^{\prime}=h \cos (\theta) x-\sqrt{h} \sin (\theta) y \\
y^{\prime}=h \sin (\theta) x+\sqrt{h} \cos (\theta) y
\end{array}\right.
$$


Then

$$
T_{h}(Q)(O)=\inf _{B^{\prime} \in \mathcal{B}_{h}} \sup _{\left(x^{\prime}, y^{\prime}\right) \in B^{\prime}}\left(Q\left(x^{\prime}, y^{\prime}\right)\right)
$$

therefore

$$
T_{h}(Q)(O)=\inf _{B \in \mathcal{B}} \inf _{0 \leq \theta \leq \pi} \sup _{(x, y) \in B}(Q(h \cos (\theta) x-\sqrt{h} \sin (\theta) y, h \sin (\theta) x+\sqrt{h} \cos (\theta) y)) .
$$

With the definition of $Q$

$$
T_{h}(Q)(O)=\inf _{B \in \mathcal{B}} \inf _{0 \leq \theta \leq \pi} \sup _{(x, y) \in B}\left(-p \sqrt{h} \sin (\theta) y+h\left(p \cos (\theta) x+a \sin ^{2}(\theta) y^{2}+b \cos ^{2}(\theta) y^{2}\right)+\mathcal{O}\left(|h|^{\frac{3}{2}}\right)\right) .
$$

It is clear that for $h$ small enough the inf-sup is reached when $\theta=0$. Then we set $\theta=0$ :

$$
T_{h}(Q)(O)=h p \inf _{B \in \mathcal{B}} \sup _{(x, y) \in B}\left(x+\frac{b}{p} y^{2}\right)+\mathcal{O}\left(h^{\frac{3}{2}}\right) .
$$

Hence

$$
T_{h}(Q)(O)=h p G\left(\frac{b}{p}\right)+\mathcal{O}\left(h^{\frac{3}{2}}\right)
$$

Since $\mathcal{B}$ is bounded, there exists a constant $M$ such that for any $B \in \mathcal{B}_{h}, B \subset D\left(O, h^{\frac{1}{2}} M\right)$. By monotonicity, we get

$$
\begin{aligned}
& T_{h}(u)(O)=T_{h}(Q)(O)+\mathcal{O}\left(|h|^{\frac{3}{2}}\right) . \\
& T_{h}(u)(O)=h p G\left(\frac{b}{p}\right)+\mathcal{O}\left(h^{\frac{3}{2}}\right) .
\end{aligned}
$$

In addition, as in Theorem 4.1, the consistency is uniform on a neighborhood of $\mathbf{x}_{o}$ on which $D u(\mathbf{x}) \neq 0$.

\section{The General CASE OF A NONDECREASING FunCtion $G$}

In this section, we prove Theorem 1.1 given in the introduction. First we recall this theorem:

Theorem 1.1. Given a function $G$ continuous, nondecreasing, there exists a family $\mathcal{B}_{\epsilon, M}, \epsilon, M>0$, such that the function defined with the alternate operator associated with $\mathcal{B}_{\epsilon, M}$

$$
u_{h, \epsilon, M}(\mathbf{x}, n h)=\left(A_{h}^{n} u\right)(\mathbf{x})
$$

tends uniformly on any compact set to the viscosity solution $u$ of

$$
\frac{\partial u}{\partial t}=|D u| G(\operatorname{curv}(u)),
$$

when $\epsilon \rightarrow 0, M \rightarrow \infty$ and $n h \rightarrow t:$

$$
\lim _{\epsilon \rightarrow 0} \lim _{M \rightarrow \infty} \lim _{n h \rightarrow t} u_{h, \epsilon, M}(\mathbf{x}, n h)=u(t, x) .
$$


Proof. 1. We shall first consider the nondecreasing function $G$ such that

$$
\left\{\begin{aligned}
\text { i) } & \exists A>0 \quad \forall x \in \mathbb{R}^{+} G^{\prime}(x) \leq A \\
\text { ii) } & \exists M>0 \quad \forall x>M \quad G(x)=G(M)+A(x-M) \\
\text { iii) } & \forall x \leq 0 \quad G(x)=0 .
\end{aligned}\right.
$$

Given $N>0$, we consider the piece of parabola $P_{s}:(x, y) \in P_{s}$ if $x=G(s)-y^{2} s$ and $-N \leq x \leq G(s)$. We call $\mathcal{B}_{N}$ the smallest isotropic set containing all the sets $P_{s}$ when $s \in[0 ; M]$. Some examples of set $P_{s}$ are given in Figure 2. In addition, $\mathcal{B}_{N}$ is a bounded family.

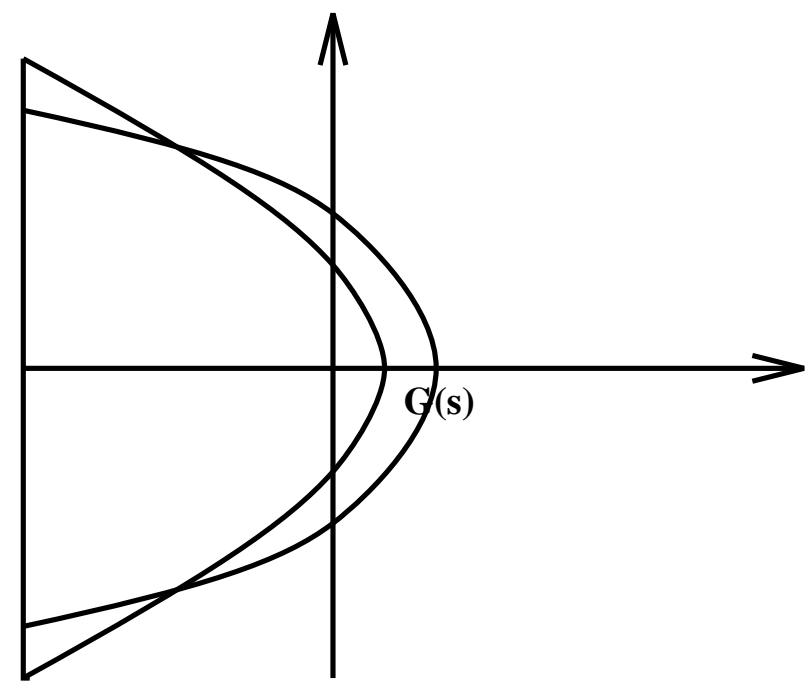

Figure 2. Examples of set $P_{s}$.

Lemma 5.1. Let $G$ defined by (2) and the family $\mathcal{B}_{N}$ as above, for any $b \in \mathbb{R}$ and for $N$ large enough

$$
G(b)=\inf _{B \in \mathcal{B}_{N}} \sup _{(x, y) \in B}\left(x+b y^{2}\right) .
$$

Proof. Given $s>0$, we define $f_{s}$ on $\mathbb{R}$ by

$$
\begin{cases}\text { If } & x \leq s \quad f_{s}(x)=G(s) \\ \text { If } & x \geq s \quad f_{s}(x)=G(s)+A(x-s) .\end{cases}
$$

As shown by Figure 3, we have for any $x \in \mathbb{R}$

We can write

$$
G(x)=\inf _{0 \leq s \leq M}\left(f_{s}(x)\right) .
$$

$$
f_{s}(x)=\sup _{0 \leq p \leq A}(G(s)+p(x-s)) .
$$

So we have

$$
G(b)=\inf _{0 \leq s \leq M}\left(\sup _{0 \leq p \leq A}(G(s)-p s+p b)\right) .
$$




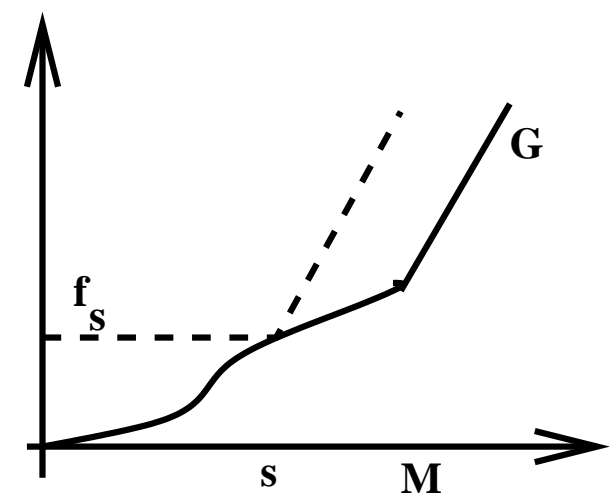

FigURE 3

We set

$$
\left\{\begin{array}{l}
x(p)=G(s)-p s \\
y(p)=\sqrt{p} .
\end{array}\right.
$$

The point $\left(x(p), y(p)\right.$ belongs to the parabola $P_{s}$. So we deduce

$$
G(b)=\inf _{0 \leq s \leq M}\left(\sup _{(x, y) \in P_{s}}\left(x+b y^{2}\right)\right) .
$$

For $N$ large enough, we have

$$
\inf _{B \in \mathcal{B}_{N}} \sup _{(x, y) \in B}\left(x+b y^{2}\right)=\inf _{0 \leq s \leq M} \sup _{(x, y) \in P_{s}}\left(x+b y^{2}\right)
$$

so we get the Lemma.

2. Lemma 5.2. Let $G$ defined by (2). The functions $u_{h}(x, n h)$, defined with the inf-sup scheme associated with $\mathcal{B}_{N}$, tend uniformly when $n h \rightarrow t$ on any compact to the viscosity solution $u(x, t)$ of

$$
\frac{\partial u}{\partial t}=|D u| G(\operatorname{curv}(u))
$$

Proof. With Theorem 4.3, we deduce that the inf-sup scheme is consistent with

$$
\frac{\partial u}{\partial t}=|D u| G(\operatorname{curv}(u))
$$

Moreover, for any $f \in \mathcal{F}(G)$, we have $f \in C^{2}([0,+\infty))$ such that $f(0)=f^{\prime}(0)=f^{\prime \prime}(0)=0$ and $f^{\prime \prime}(r)>0$ for $r>0$. With a Taylor expansion, we get

$$
f\left(\left|x-x_{o}\right|\right)=\mathcal{O}\left(\left|x-x_{o}\right|^{3}\right) .
$$

With the definition of $\mathcal{B}_{h}$, we have

$$
T_{h} f\left(\left|\cdot-x_{o}\right|\right)\left(x_{o}\right)=\mathcal{O}\left(h^{\frac{3}{2}}\right) .
$$


Thus

$$
\lim _{h \rightarrow 0} \frac{T_{h} f\left(\left|.-x_{o}\right|\right)\left(x_{o}\right)}{h}=0 .
$$

Thus, with Theorem 3.6, we deduce the Lemma.

3. Thus, given a nondecreasing continuous function $G$, let be $\epsilon>0$, we note $f_{s}^{\epsilon}$ the function defined on $\mathbb{R}$ by

$$
\left\{\begin{array}{lll}
\text { If } & x \leq s & f_{s}^{\epsilon}(x)=G(s) \\
\text { If } & x \geq s & f_{s}^{\epsilon}(x)=G(s)+\frac{1}{\epsilon}(x-s)
\end{array} .\right.
$$

We set

$$
G_{\epsilon}(x)=\inf _{s \geq 0}\left(f_{s}^{\epsilon}(x)\right) .
$$

Lemma 5.3. Let $u_{\epsilon}$ be the viscosity solution of

$$
\frac{\partial u_{\epsilon}}{\partial t}=\left|D u_{\epsilon}\right| G_{\epsilon}\left(\operatorname{curv}\left(u_{\epsilon}\right)\right),
$$

then $u_{\epsilon} \rightarrow u$ when $\epsilon \rightarrow 0$ and $u$ is the viscosity solution of

$$
\frac{\partial u}{\partial t}=|D u| G(\operatorname{curv}(u)) .
$$

Proof. We only have to prove that the functions $G_{\epsilon}$ satisfy the assumptions of Theorem 3.4.

The functions $G_{\epsilon}$ converge uniformly locally to $G$. Let be $f \in \mathcal{F}(G)$, then

$$
\lim _{p \rightarrow 0} f^{\prime}(|p|) G\left(\frac{-1}{|p|}\right)=\lim _{p \rightarrow 0} f^{\prime}(|p|) G\left(\frac{1}{|p|}\right)=0 .
$$

When $p$ is small enough, we have

$$
0 \leq f^{\prime}(|p|) G_{\epsilon}\left(\frac{1}{|p|}\right) \leq f^{\prime}(|p|) G\left(\frac{1}{|p|}\right)
$$

hence

$$
\lim _{p \rightarrow 0} f^{\prime}(|p|) G_{\epsilon}\left(\frac{1}{|p|}\right)=0 .
$$

In addition, $G_{\epsilon}\left(\frac{-1}{|p|}\right)=0$. Finally, let be $f \in \mathcal{F}(G)$, as $f^{\prime}(|p|) G_{\epsilon}\left(\frac{1}{|p|}\right) \geq 0$ and $f^{\prime}(|p|) G_{\epsilon}\left(\frac{-1}{|p|}\right)=0$, we deduce the last needed condition to apply the above theorem.

4. It remains to approach $u_{\epsilon}$. Again, given $\epsilon>0$, we approximate $G_{\epsilon}$ by $G_{\epsilon}^{M}$ with $M>0$ and

$$
\left\{\begin{array}{lll}
\text { If } & x \leq M & G_{\epsilon}^{M}(x)=G_{\epsilon}(x) \\
\text { If } & x \geq M & G_{\epsilon}^{M}(x)=G(M)+\frac{1}{\epsilon}(x-M) .
\end{array}\right.
$$

It is easy to check the assumptions of Theorem 3.4, then

Lemma 5.4. Let $u_{M}$ be the viscosity solution of

$$
\frac{\partial u_{M}}{\partial t}=\left|D u_{M}\right| G_{\epsilon}^{M}\left(\operatorname{curv}\left(u_{M}\right)\right)
$$


then $u_{M} \rightarrow u$ when $M \rightarrow \infty$ and $u$ is the viscosity solution of

$$
\frac{\partial u}{\partial t}=|D u| G_{\epsilon}(\operatorname{curv}(u))
$$

5. Let $\epsilon, M>0$ be two parameters. We consider the function $G_{\epsilon}^{M}$ defined as in step 4. Since $G_{\epsilon}^{M}$ satisfies the assumptions of Lemma 5.2, step 2, we can construct a family $\mathcal{B}_{\epsilon}^{M}$ such that the associated functions $u_{\epsilon, h}^{M}(x, n h)$ tends uniformly on any compact set to the viscosity solution $u_{\epsilon}^{M}$ of

$$
\frac{\partial u}{\partial t}=|D u| G_{\epsilon}^{M}(\operatorname{curv}(u)) .
$$

With Lemma 5.4, we let $M$ tend to $\infty$, then $u_{\epsilon}^{M}$ tends uniformly on any compact set to the viscosity solution $u_{\epsilon}$ of

$$
\frac{\partial u}{\partial t}=|D u| G_{\epsilon}(\operatorname{curv}(u))
$$

With Lemma 5.3, we let $\epsilon$ tend to 0 , then $u_{\epsilon}$ tend uniformly on any compact set to the viscosity solution $u$ of

$$
\frac{\partial u}{\partial t}=|D u| G(\operatorname{curv}(u)) .
$$

\section{CASE Where $G(s)=s^{\gamma}$}

\subsection{Notations}

The approach is simpler than in the preceding case because of the concavity or convexity of $G$. We use the first consistency theorem about the asymptotic behaviour of the scheme $T_{h}$ when $\mathcal{B}_{h}=h \mathcal{B}$. We adopt the following notations. Let be $a=\left(\frac{\gamma^{1 / 2}}{1-\gamma}\right)^{\frac{2}{\gamma+1}}$. We set $I=[0 ; a]$ and consider the curve $C=(x(t), y(t))$ when $t \in I$ defined by

$$
\left\{\begin{array}{l}
x(t)=(1-\gamma) t^{\gamma} \\
y(t)=(\gamma)^{\frac{1}{2}} t^{\frac{\gamma-1}{2}}
\end{array}\right.
$$

The equation of $C$ is

$$
y=\gamma\left(\frac{-1}{1-\gamma}\right)^{\frac{\gamma-1}{2 \gamma}}(-x)^{\frac{\gamma-1}{2 \gamma}} .
$$

The exponent of $x$ belongs to the interval $\left[0 ; \frac{1}{2}\left[\right.\right.$. Let $B_{t}$ be the set formed by the four points

$$
B_{t}=\{(x(t), y(t)) ;(x(t),-y(t)) ;(-x(t), y(t)) ;(-x(t),-y(t))\}
$$

We call $\mathcal{B}_{1}$ the smallest isotropic set containing all the sets $B_{t}$ when $t \in I$. We notice that the element containing only the origin belongs to $\mathcal{B}_{1}$. Let $R_{t}$ be the rectangle the vertices of which are the points of $B_{t}$. We call $\mathcal{B}_{2}$ the smallest isotropic set containing all the sets $R_{t}$ when $t \in I$.

Theorem 6.1. Consider the family $\mathcal{B}=\mathcal{B}_{1}$ or $=\mathcal{B}_{2}$ just defined above. Set $I S_{h}, S I_{h}$ and $A_{h}$ the operators defined by

$$
\begin{aligned}
& \left(I S_{h} u\right)(\mathbf{x})=\inf _{B \in h \mathcal{B}} \sup _{\mathbf{y} \in \mathbf{x}+B} u(\mathbf{y}), \\
& \left(S I_{h} u\right)(\mathbf{x})=\sup _{B \in h \mathcal{B}} \inf _{\mathbf{y} \in \mathbf{x}+B} u(\mathbf{y}),
\end{aligned}
$$




$$
\left(A_{h} u\right)(\mathbf{x})=\left(I S_{h} \circ S I_{h} u\right)(\mathbf{x}) .
$$

If $\gamma \in[0 ; 1]$ and $\mathcal{B}=\mathcal{B}_{2}$, the functions $u_{h}(\mathbf{x}, n h)$, defined with the inf-sup scheme $A_{h}$, tend uniformly when $n h \rightarrow t$ on any compact set to the viscosity solution $u(\mathbf{x}, t)$ of

$$
\frac{\partial u}{\partial t}=|D u|(\operatorname{curv}(u))^{\gamma}
$$

If $\gamma \in[1 ;+\infty]$ and $\mathcal{B}=\mathcal{B}_{1}$, the functions $u_{h}(\mathbf{x}, n h)$, defined with the inf-sup scheme $A_{h}$, tend uniformly when $n h \rightarrow t$ on any compact set to the viscosity solution $u(\mathbf{x}, t)$ of

$$
\frac{\partial u}{\partial t}=|D u|(\operatorname{curv}(u))^{\gamma}
$$

\section{2. $\gamma$ is larger than 1}

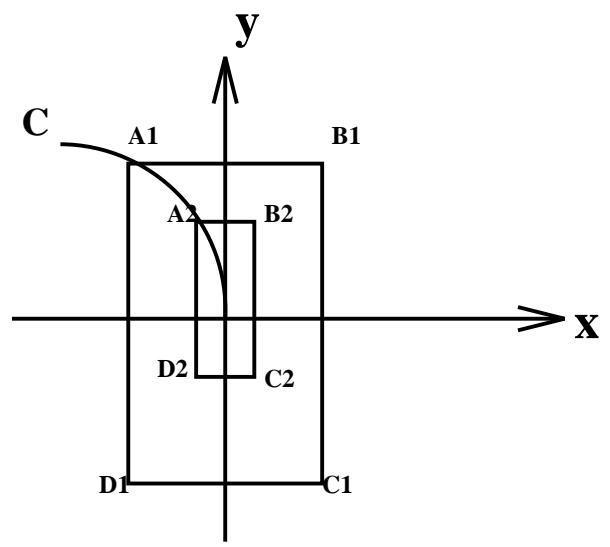

Figure 4 . The four corners of the rectangle $(A 1, B 1, C 1, D 1)$ constitute an element of $\mathcal{B}_{1}$. In the same way the four corners of the rectangle $(A 2, B 2, C 2, D 2)$.

Let us first prove Theorem 6.1 when $\gamma$ is larger than 1.

1. Lemma 6.2.

- For all $b$ in $I, \sup _{\mathcal{B}_{1}} \inf _{B}\left(x+b y^{2}\right)=b^{\gamma}$,

- for all $b \leq 0, \sup _{\mathcal{B}_{1}} \inf _{B}\left(x+b y^{2}\right)=0$.

Proof. First we assume $b \in I$. We call $B_{t}^{\theta}$ the image of $B_{t}$ by the rotation centered in $\mathrm{O}$ with angle $\theta$. Because of the symmetry of $B_{t}$, we restrict $\theta$ to $\left[0 ; \frac{\pi}{2}\right]$. So we have

$$
\sup _{\mathcal{B}_{1}} \inf _{B}\left(x+b y^{2}\right)=\sup _{t \in I} \sup _{\theta} \inf _{B_{t}^{\theta}}\left(x+b y^{2}\right) \text {. }
$$

We consider the point $A_{t}=(x(t), y(t))$. As $t \in I$, we have $|x(t)| \leq y(t)$, we deduce that for all $\theta \in\left[0 ; \frac{\pi}{2}\right]$

$$
\inf _{B_{t}^{\theta}}\left(x+b y^{2}\right) \leq \inf _{B_{t}^{0}}\left(x+b y^{2}\right)
$$

therefore

$$
\sup _{\theta} \inf _{B_{t}^{\theta}}\left(x+b y^{2}\right)=\inf _{B_{t}^{0}}\left(x+b y^{2}\right) .
$$


Finally we get

$$
\sup _{\mathcal{B}_{1}} \inf _{B}\left(x+b y^{2}\right)=\sup _{t \in I} \inf _{B_{t}^{0}}\left(x+b y^{2}\right) .
$$

It remains to prove that

$$
\sup _{t \in I} \inf _{B_{t}^{0}}\left(x+b y^{2}\right)=b^{\gamma}
$$

By construction we have

$$
\sup _{t \in I} \inf _{B_{t}}\left(x+b y^{2}\right)=\sup _{t \in I}\left(x(t)+b y(t)^{2}\right) .
$$

On the other hand, since $G(s)=s^{\gamma}$ is a convex function on $I, G$ is the supremum of its tangent lines. Thus, for any $s$ in $I$

$$
G(s)=\sup _{t \in I}\left(G(t)+G^{\prime}(t)(s-t)\right)
$$

or

$$
G(s)=\sup _{t \in I}\left(x(t)+s y(t)^{2}\right) .
$$

From this and the definition of $B_{t}$, we get (4).

We now assume $b \leq 0$. For all $\theta$, it is posssible to find a point $(x, y) \in B_{t}^{\theta}$ with $x \leq 0$. Thus,

$$
\inf _{B_{t}^{\theta}}\left(x+b y^{2}\right) \leq 0
$$

In addition, there is only one point in $B_{0}$ : the origin, so

$$
\inf _{B_{0}^{\theta}}\left(x+b y^{2}\right)=0
$$

Finally,

$$
\sup _{t \in I} \inf _{B_{t}}\left(x+b y^{2}\right)=0
$$

2. We have a dual lemma with the inf-sup scheme, since

$$
\inf _{\mathcal{B}_{1}} \sup _{B}(-u)=-\sup _{\mathcal{B}_{1}} \inf _{B}(u)
$$

So we can also deduce

\section{Lemma 6.3.}

- for all $b \geq 0, \inf _{\mathcal{B}_{1}} \sup _{B}\left(x+b y^{2}\right)=0$,

- for all $b$ of $I, \inf _{\mathcal{B}_{1}} \sup _{B}\left(x+b y^{2}\right)=(-b)^{\gamma}$.

3. Since the family $\mathcal{B}_{1}$ is bounded, isotropic and each element is symmetric with respect to O, we deduce with Theorem 4.1 that for any function $u \in C^{\gamma+2}\left(\mathbb{R}^{2}\right)$ in a neighborhood of $\mathbf{x}_{o} \in \mathbb{R}^{2}$, if $\left|D u\left(\mathbf{x}_{o}\right)\right| \neq 0$

$$
\begin{aligned}
& \lim _{h \rightarrow 0} \frac{I S_{h} u\left(\mathbf{x}_{o}\right)-u\left(\mathbf{x}_{o}\right)}{h^{\gamma+1}}=\left|D u\left(\mathbf{x}_{o}\right)\right| G\left(\operatorname{curv}(u)^{+}\left(\mathbf{x}_{o}\right)\right), \\
& \lim _{h \rightarrow 0} \frac{S I_{h} u\left(\mathbf{x}_{o}\right)-u\left(\mathbf{x}_{o}\right)}{h^{\gamma+1}}=\left|D u\left(\mathbf{x}_{o}\right)\right| G\left(\operatorname{curv}(u)^{-}\left(\mathbf{x}_{o}\right)\right),
\end{aligned}
$$




$$
\lim _{h \rightarrow 0} \frac{A_{h} u\left(\mathbf{x}_{o}\right)-u\left(\mathbf{x}_{o}\right)}{h^{\gamma+1}}=\left|D u\left(\mathbf{x}_{o}\right)\right| G\left(\operatorname{curv}(u)\left(\mathbf{x}_{o}\right)\right),
$$

with $(\operatorname{curv} u)^{+}=\max (\operatorname{curv} u, 0),(\operatorname{curv} u)^{-}=\max (-\operatorname{curv} u, 0)$, and

$$
G(r)=r^{\gamma} .
$$

In addition, the consistency is uniform.

Then the scheme $A_{h}$ is uniformly consistent with $\frac{\partial u}{\partial t}=|D u| G(\operatorname{curv}(u))$.

4. It is easy to check that for any $f \in \mathcal{F}(G)$, for any $\mathbf{x}_{o}$,

$$
\lim _{h \rightarrow 0} \frac{A_{h} f\left(\left|.-\mathbf{x}_{o}\right|\right)\left(\mathbf{x}_{o}\right)}{h}=0 .
$$

In addition, $A_{h}$ is monotone, stable and commuting with the addition of constants $\left(i . e . T_{h}(u+C)=\right.$ $\left.T_{h} u+C\right)$. Thus, we can apply Theorem 3.6 and complete the proof.

\section{3. $\gamma$ is smaller than 1}

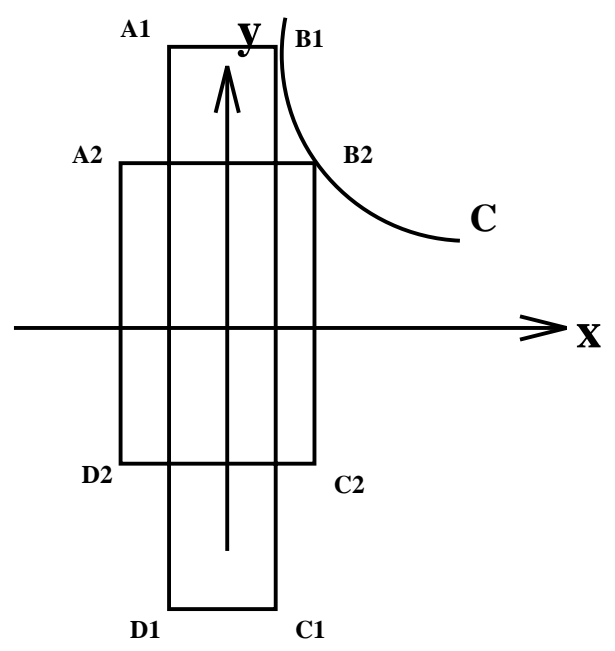

Figure 5. The rectangle $(A 1, B 1, C 1, D 1)$ constitutes an element of $\mathcal{B}_{2}$. In the same way the rectangle $(A 2, B 2, C 2, D 2)$.

We prove Theorem 6.1 if $\gamma$ is smaller than 1.

1. We prove the consistency result for $I S_{h}(u)$, and thanks to the relation

$$
\sup _{\mathcal{B}_{2}} \inf _{B}(u(\mathbf{y}))=-\inf _{\mathcal{B}_{2}} \sup _{B}(-u(\mathbf{y}))
$$

we deduce the result for $S I_{h}$, and then for $C_{h}(u)$ and $A_{h}$. Since the family $\mathcal{B}_{2}$ is not bounded, that is to say there is no disk containing every element of $\mathcal{B}_{2}$, the value of $I S_{h} u(\mathbf{x})$ depends on values of $u(\mathbf{y})$ at points $\mathbf{y}$ arbitrarily far from $\mathbf{x}$. Therefore, this operator looks like an a priori non local operator no matter how small $h$ is. Nevertheless, we shall show that the behaviour of $I S_{h}$ is very close to an operator defined with a bounded family. We defined this new family $\mathcal{B}_{\epsilon}$ by restricting the domain of $t$ to $[\epsilon ; a]$ instead of $[0 ; a]$ with $\epsilon>0$. This new family is bounded, isotropic and each element $B \in \mathcal{B}_{\epsilon}$ is symmetric 
with respect to O. Therefore, we can apply Theorem 4.1 about the asymptotic behaviour to $I S_{\epsilon}$ deduced from the bounded family $\mathcal{B}_{\epsilon}$

$$
I S_{h, \epsilon} u\left(\mathbf{x}_{o}\right)=u\left(\mathbf{x}_{o}\right)+h|D u| G_{\epsilon}(h \operatorname{curv}(u))+K_{\epsilon, \mathbf{x}_{o}} h^{2+1 / 3}
$$

and

$$
G_{\epsilon}(b)=\inf _{\mathcal{B}_{\epsilon}} \sup _{B}\left(x+b y^{2}\right)=\inf _{t \in[\epsilon ; a]} \sup _{B_{t}}\left(x+b y^{2}\right) .
$$

So the idea is to let $\epsilon$ tend to 0 . First, we compute $G(b)=\inf _{\mathcal{B}_{2}} \sup _{B}\left(x+b y^{2}\right)$ and we deduce $G_{\epsilon}(b)$. Then, by using the locality lemma, we compare $I S_{h}$ and $I S_{h \epsilon}$.

2. Lemma 6.4.

- If $b \in I, \inf _{\mathcal{B}_{2}} \sup _{B}\left(x+b y^{2}\right)=b^{\gamma}$,

- if $b \leq 0 \inf _{\mathcal{B}_{2}} \sup _{B}\left(x+b y^{2}\right)=0$.

Proof. First, we assume $b \in I$. We call $B_{t}^{\theta}$ the image of $B_{t}$ by a rotation of $\theta$. Because of the symmetry of $B_{t}$, we restrict $\theta$ to $\left[0 ; \frac{\pi}{2}\right]$. So we have

$$
\inf _{\mathcal{B}_{2}} \sup _{B}\left(x+b y^{2}\right)=\inf _{t \in I} \inf _{\theta} \sup _{B_{t}^{\theta}}\left(x+b y^{2}\right) .
$$

We consider the point $A_{t}=(x(t), y(t))$. Since we have $|x(t)| \leq y(t)$, we deduce that for all $\theta \in\left[0 ; \frac{\pi}{2}\right]$

$$
\sup _{B_{t}^{\theta}}\left(x+b y^{2}\right) \geq \sup _{B_{t}^{0}}\left(x+b y^{2}\right)
$$

therefore

$$
\inf _{\theta} \sup _{B_{t}^{\theta}}\left(x+b y^{2}\right)=\sup _{B_{t}^{0}}\left(x+b y^{2}\right)
$$

and finally we get

$$
\inf _{\mathcal{B}_{2}} \sup _{B}\left(x+b y^{2}\right)=\inf _{t \in I} \sup _{B_{t}^{0}}\left(x+b y^{2}\right) .
$$

It remains to prove

$$
\inf _{t \in I} \sup _{B_{t}^{0}}\left(x+b y^{2}\right)=b^{\gamma} .
$$

Now, since $G(s)=s^{\gamma}$ is a concave function on $I, G$ is the infimum of its tangent lines. Thus, for any $s$ in $I$

or

$$
G(s)=\inf _{t \in I}\left(G(t)+G^{\prime}(t)(s-t)\right)
$$

$$
G(s)=\inf _{t \in I}\left(x(t)+s y(t)^{2}\right)
$$

From this we get (5).

We now assume $b \leq 0$. So we have $x+b y^{2} \leq x$. Then, since the origin belongs to any set $B$ of $\mathcal{B}_{2}$, we have

therefore,

$$
0 \leq \sup _{B}\left(x+b y^{2}\right) \leq \sup _{B}(x)
$$

$$
0 \leq \inf _{B \in \mathcal{B}_{2}} \sup _{B}\left(x+b y^{2}\right) \leq \inf _{B \in \mathcal{B}_{2}} \sup _{B}(x)=0
$$


Finally,

$$
\inf _{B \in \mathcal{B}_{2}} \sup _{B}\left(x+b y^{2}\right)=0 .
$$

3. We have a dual lemma with sup-inf scheme since

$$
\inf _{\mathcal{B}_{2}} \sup _{B}(-u)=-\sup _{\mathcal{B}_{2}} \inf _{B}(u) .
$$

Lemma 6.5.

- For all $b \geq 0, \sup _{\mathcal{B}_{2}} \inf _{B}\left(x+b y^{2}\right)=0$,

- for all $b$ of $I, \sup _{\mathcal{B}_{2}} \inf _{B}\left(x+b y^{2}\right)=(-b)^{\gamma}$.

4. Then, it is easy to compute $G_{\epsilon}$.

$$
\left\{\begin{array}{lll}
\text { If } & x \geq \epsilon & G_{\epsilon}(x)=G(s) \\
\text { If } & \epsilon \geq x \geq 0 & G_{\epsilon}(x)=G(\epsilon)+G^{\prime}(\epsilon)(x-\epsilon) \\
\text { If } & 0 \geq x & G_{\epsilon}(x)=G(\epsilon)-\epsilon G^{\prime}(\epsilon) .
\end{array}\right.
$$

We get the following graphics for the two functions $G$ and $G_{\epsilon}$ :

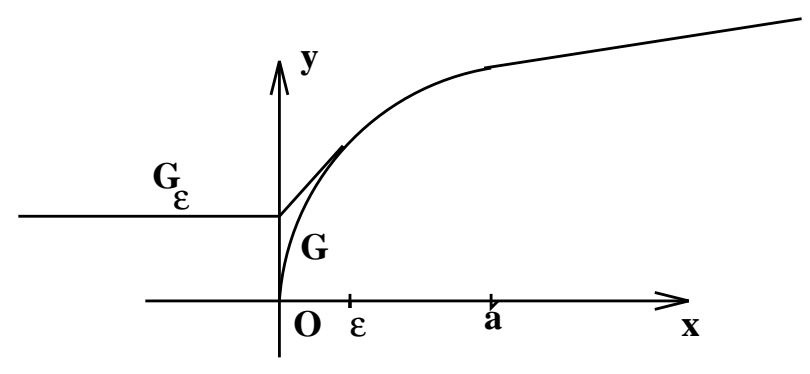

Figure $6 . G$ and $G_{\epsilon}$.

So

$$
\left|G(b)-G_{\epsilon}(b)\right| \leq x(\epsilon)=(1-\gamma) \epsilon^{\gamma} .
$$

5. The next lemma expresses that the families $\mathcal{B}_{2}$ and $\mathcal{B}_{\epsilon}$ are very close for the semi-Hausdorff distance. We introduce the Hausdorff semi-distance for two bounded sets of $\mathbb{R}^{2}$

$$
\delta\left(B^{\prime}, B\right)=\sup _{x \in B^{\prime}}\left(\inf _{y \in B}|x-y|\right) .
$$

\section{Lemma 6.6. Locality}

Let $\epsilon>0$, and $r=h\left(x^{2}(\epsilon)+y^{2}(\epsilon)\right)^{\frac{1}{2}}$. Then for every $B \in h \mathcal{B}_{2}$, there exists $B^{\prime} \in \mathcal{B}_{\epsilon h}$, such that

$$
\delta\left(B^{\prime}, B \cap D(0, r)\right) \leq(1-\gamma) h \epsilon^{\gamma} .
$$

Proof. If $B \in \mathcal{B}_{\epsilon h}$, we choose for $B^{\prime}$ the set $B$. Assume now that $B$ doesn't belong to the family $\mathcal{B}_{\epsilon h}$. We choose $\vec{i}$ so that this vector is parallel to the smaller side of the rectangle $B$, and $\vec{j}$ is orthogonal to $\vec{i}$. We consider the element $B^{\prime}$ of $\mathcal{B}_{\epsilon h}$ like in Figure 7 . Then we have

$$
\delta\left(B^{\prime}, B \cap D(0, r)\right) \leq h x_{\epsilon}=(1-\gamma) \epsilon^{\gamma} h .
$$




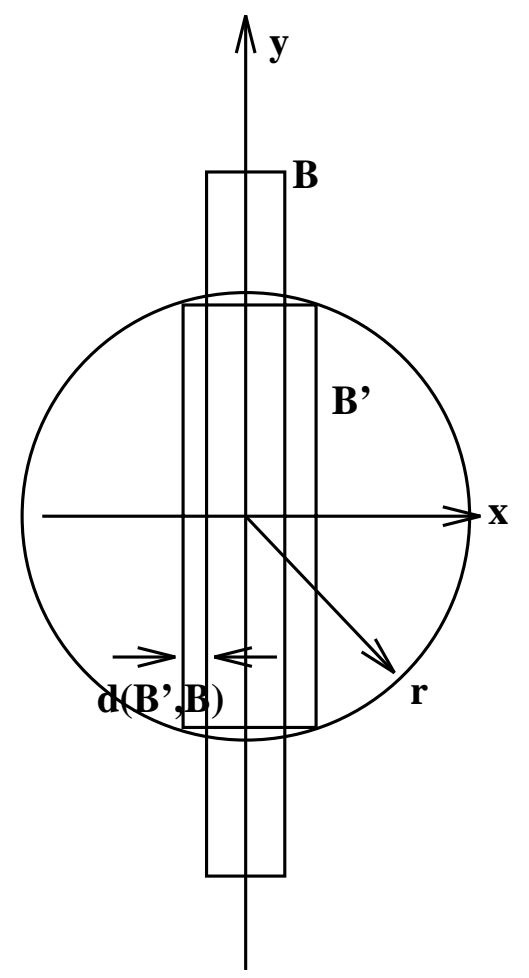

Figure $7 . B$ and $B^{\prime}$ are very close for the semi Hausdorff distance.

6. Lemma 6.7. Local Monotonicity

Let $\epsilon>0$ and $r=h\left(x^{2}(\epsilon)+y^{2}(\epsilon)\right)^{\frac{1}{2}}$. Let $u$ and $v$ be two $K$-Lipschitz functions such that for every $\mathbf{x} \in D\left(\mathbf{x}_{o}, r\right)$

Then we have

$$
u(\mathbf{x}) \geq v(\mathbf{x}) .
$$

$$
I S_{h} u \geq I S_{h} v-K(1-\gamma) h \epsilon^{\gamma}
$$

and

$$
I S_{h \epsilon} u(\mathbf{y}) \geq I S_{h} u(\mathbf{y}) \geq I S_{h \epsilon} u(\mathbf{y})-K(1-\gamma) h \epsilon^{\gamma} .
$$

Proof. We have

$$
I S_{h} u\left(\mathbf{x}_{o}\right) \geq \inf _{B \in h \mathcal{B}_{2}+\mathbf{x}_{o}} \sup _{\mathbf{y} \in B \cap D\left(\mathbf{x}_{o}, r\right)} u(\mathbf{y}) \geq \inf _{B \in h \mathcal{B}_{2}+\mathbf{x}_{o}} \sup _{\mathbf{y} \in B \cap D\left(\mathbf{x}_{o}, r\right)} v(\mathbf{y}) .
$$

Let $B \in h \mathcal{B}_{2}$, we consider the $B^{\prime}$ obtained with the preceding lemma. As $v$ is K-Lipschitz, we have for $\mathbf{y} \in B^{\prime}$ and $\mathbf{x} \in B \cap D$

$$
v(\mathbf{y}) \leq v(\mathbf{x})+K|\mathbf{y}-\mathbf{x}|,
$$

therefore,

so,

$$
v(\mathbf{y}) \leq \sup _{\mathbf{x} \in B \cap D} v(\mathbf{x})+K \inf _{\mathbf{x} \in B \cap D}|\mathbf{y}-\mathbf{x}|,
$$

$$
\sup _{B^{\prime}} v(\mathbf{y}) \leq \sup _{\mathbf{x} \in B \cap D} v(\mathbf{x})+K \delta\left(B^{\prime}, B \cap D\right),
$$


thus,

we deduce

$$
\sup _{B^{\prime}} v(\mathbf{y}) \leq \sup _{\mathbf{x} \in B \cap D} v(\mathbf{x})+K(1-\gamma) h \epsilon^{\gamma}
$$

$$
I S_{h} u\left(\mathbf{x}_{o}\right) \geq \inf _{B \in h \mathcal{B}_{2}+\mathbf{x}_{o}} \sup _{\mathbf{y} \in B^{\prime}} v(\mathbf{y})-K(1-\gamma) h \epsilon^{\gamma} I S_{h \epsilon} v(\mathbf{y})-K(1-\gamma) h \epsilon^{\gamma} .
$$

As $\mathcal{B}_{\epsilon h} \subset h \mathcal{B}_{2}$, we have

$$
I S_{h \epsilon} v(\mathbf{y}) \geq I S_{h} v(\mathbf{y})
$$

7. So we have the relation

$$
I S_{h, \epsilon} u\left(\mathbf{x}_{o}\right)=u\left(\mathbf{x}_{o}\right)+h|D u| G_{\epsilon}(h \operatorname{curv} u)+K_{\epsilon, \mathbf{x}_{o}} h^{2+1 / 3} .
$$

We set

$$
\epsilon=h^{\omega} .
$$

With the preceding results, we have

$$
I S_{h, \epsilon} u\left(\mathbf{x}_{o}\right)=I S_{h} u\left(\mathbf{x}_{o}\right)+K_{1, \epsilon, \mathbf{x}_{o}} h^{1+\omega \gamma},
$$

and

We deduce

$$
G_{\epsilon}(h \operatorname{curv} u)=G(h \operatorname{curv} u)+K_{2, \epsilon, \mathbf{x}_{o}} h^{\omega \gamma} .
$$

$$
I S_{h} u\left(\mathbf{x}_{o}\right)=u\left(\mathbf{x}_{o}\right)+h|D u| G(h \operatorname{curv} u)+K_{3, \epsilon, \mathbf{x}_{o}} h^{1+\omega \gamma}+K_{\epsilon, \mathbf{x}_{o}} h^{2+1 / 3} .
$$

If $\operatorname{curv}(u)\left(\mathbf{x}_{o}\right)<0$, then

$$
I S_{h} u\left(\mathbf{x}_{o}\right)=u\left(\mathbf{x}_{o}\right)+K_{3, \epsilon, \mathbf{x}_{o}} h^{1+\omega \gamma}+K_{\epsilon, \mathbf{x}_{o}} h^{2+1 / 3} .
$$

If $\operatorname{curv}(u)\left(\mathbf{x}_{o}\right)>0$, then

$$
I S_{h} u\left(\mathbf{x}_{o}\right)=u\left(\mathbf{x}_{o}\right)+h^{\gamma+1}|D u|(\operatorname{curv} u)^{\gamma}+K_{3, \epsilon, \mathbf{x}_{o}} h^{1+\omega \gamma}+K_{\epsilon, \mathbf{x}_{o}} h^{2+1 / 3} .
$$

In order to get the consistency result, it is enough to choose $\omega>1$.

Moreover, we can prove that the consistency is uniform on any compact set on which $|D u|(\mathbf{x}) \neq 0$ if $\omega<2$. To get this result, we only have to prove that the family $\mathcal{B}_{h \epsilon}$ remains included in a ball whose radius tends to 0 with $h$. For all $B \in \mathcal{B}_{h \epsilon}, B$ is included in a ball with radius $r=h\left(x^{2}(\epsilon)+y^{2}(\epsilon)\right)^{1 / 2}$. An equivalent of $r$ in $h=0$ is $h^{1+\omega \frac{\gamma-1}{2}}$. Then, with the condition $\omega<2, r$ tends to 0 with $h$. Therefore the constants $K_{\epsilon, \mathbf{x}_{o}}$ and $K_{3, \epsilon, \mathbf{x}_{o}}$ are bounded, so we get the uniform consistency.

Then the scheme $A_{h}$ is uniformly consistent with $\frac{\partial u}{\partial t}=|D u| G(\operatorname{curv}(u))$ and

$$
G(r)=r^{\gamma} .
$$

8. It is easy to check that for any $f \in \mathcal{F}(G)$, for any $\mathbf{x}_{o}$,

$$
\lim _{h \rightarrow 0} \frac{A_{h} f\left(\left|.-\mathbf{x}_{o}\right|\right)\left(\mathbf{x}_{o}\right)}{h}=0 .
$$

In addition, $A_{h}$ is monotone, stable and commuting with the addition of constants $\left(i . e . T_{h}(u+C)=\right.$ $\left.T_{h} u+C\right)$. Thus, we can apply Theorem 3.6 and complete the proof. 


\section{Appendix}

Proof of Theorem 3.6. 1. We define

$$
u^{*}(\mathbf{x}, t)=\limsup _{n h \rightarrow t, h \rightarrow 0} u_{h}(\mathbf{x}, n h)
$$

and

$$
u_{*}(\mathbf{x}, t)=\liminf _{n h \rightarrow t, h \rightarrow 0} u_{h}(\mathbf{x}, n h) .
$$

Next we prove that $u^{*}$ is a usc subsolution and $u_{*}$ is a lsc supersolution. As $u^{*}(\mathbf{x}, 0)=u_{*}(\mathbf{x}, 0)$, we deduce with the comparison principle $[9] u^{*}(\mathbf{x}, t) \leq u_{*}(\mathbf{x}, t)$. But the opposite inequality is obvious, so we get the equality which means the convergence of the scheme.

2. We only prove that the function $u^{*}$ is a subsolution since the argument for $u_{*}$ is identical. Let $\phi$ be an admissible function and $\left(\mathbf{x}_{o}, t_{o}\right)$ be a local maximum of $u-\phi$ on $\bar{\Omega}$. We set $D=D\left(\left(\mathbf{x}_{o}, t_{o}\right), r\right)$ such that $\left(\mathbf{x}_{o}, t_{o}\right)$ is a strict local maximum of $u-\phi$ on $D$. With standard arguments, it is possible to extract a sequence $\left(\mathbf{x}_{h}, n_{h} h\right) \rightarrow\left(\mathbf{x}_{o}, t_{o}\right)$ when $h \rightarrow 0$ and $\left(\mathbf{x}_{h}, n_{h} h\right)$ is a local maximum of $u_{h}-\phi$ on $D$. Then, for $h$ small enough and for any $\mathbf{y} \in D\left(\mathbf{x}, \frac{r}{2}\right)$, we get $\left(\mathbf{y},\left(n_{h}-1\right) h\right) \in D$. Hence

$$
u_{h}\left(\mathbf{y},\left(n_{h}-1\right) h\right) \leq u_{h}\left(\mathbf{x}_{h}, n_{h} h\right)-\phi\left(\mathbf{x}_{h}, n_{h} h\right)+\phi\left(\mathbf{y},\left(n_{h}-1\right) h\right)
$$

With the local comparison principle, and the commutation with the addition of constant we deduce

$$
T_{h} u_{h}\left(.,\left(n_{h}-1\right) h\right)\left(\mathbf{x}_{h}\right) \leq u_{h}\left(\mathbf{x}_{h}, n_{h} h\right)-\phi\left(\mathbf{x}_{h}, n_{h} h\right)+T_{h} \phi\left(.,\left(n_{h}-1\right) h\right)\left(\mathbf{x}_{h}\right)+o(h) .
$$

With standard arguments, we get the following lemma.

Lemma 7.1. If $u$ is a subsolution (resp. supersolution) for any function $\phi$ admissible $C^{\infty}$ with $\phi(\mathbf{x}, t)$ $=f(\mathbf{x})+g(t)$, then $u$ is a subsolution (resp. a supersolution).

Then we set $\phi(\mathbf{x}, t)=f(\mathbf{x})+g(t)$ in $(6)$, we get

$$
u_{h}\left(\mathbf{x}_{h}, n_{h} h\right) \leq u_{h}\left(\mathbf{x}_{h}, n_{h} h\right)-f\left(\mathbf{x}_{h}\right)-g\left(n_{h} h\right)+T_{h} f\left(\mathbf{x}_{h}\right)+g\left(\left(n_{h}-1\right) h\right)+o(h),
$$

therefore,

$$
g\left(n_{h} h\right)-g\left(\left(n_{h}-1\right) h\right)+f\left(\mathbf{x}_{h}\right)-T_{h} f\left(\mathbf{x}_{h}\right)+o(h) \leq 0
$$

3. First, we consider the case $|D \phi|\left(\mathbf{x}_{o}, t_{o}\right)=|D f|\left(\mathbf{x}_{o}\right) \neq 0$. Then, for $h$ small enough, $|D f|\left(\mathbf{x}_{h}\right) \neq 0$. We conclude with the consistancy property

$$
h g^{\prime}(c)-h\left|D f\left(\mathbf{x}_{h}\right)\right| G\left(\operatorname{curv}\left(f\left(\mathbf{x}_{h}\right)\right)\right)+o(h) \leq 0
$$

with $c \in\left[\left(n_{h}-1\right) h ; n_{h} h\right]$. We divide by $h$ and we let $h$ tend to 0 , as $G$ is continuous, we get

$$
g^{\prime}\left(t_{o}\right)-\left|D f\left(\mathbf{x}_{o}\right)\right| G\left(\operatorname{curv}\left(f\left(\mathbf{x}_{o}\right)\right)\right) \leq 0
$$

4. We consider now the case $|D \phi|\left(\mathbf{x}_{o}, t_{o}\right)=0$. Since $\phi$ is admissible, there exists a constant $\delta>0$ and functions $f \in \mathcal{F}(G)$ and $\omega \in C((0,+\infty))$ with $\lim _{r \rightarrow 0} \frac{\omega(r)}{r}=0$ such that

$$
\left|\phi(\mathbf{x}, t)-\phi\left(\mathbf{x}_{o}, t_{o}\right)-\phi_{t}\left(\mathbf{x}_{o}, t_{o}\right)\left(t-t_{o}\right)\right| \leq f\left(\left|\mathbf{x}-\mathbf{x}_{o}\right|\right)+w\left(\left|t-t_{o}\right|\right)
$$


for any $(\mathbf{x}, t) \in B\left(\left(\mathbf{x}_{o}, t_{o}\right), \delta\right)$.

We assume, as [9], that $\omega \in C^{1}((0,+\infty))$ and $\omega(0)=\omega^{\prime}(0)=0$ and $\omega(r)>0$ for $r \neq 0$. We set

$$
\psi(\mathbf{x}, t)=\phi_{t}\left(\mathbf{x}_{o}, t_{o}\right)\left(t-t_{o}\right)+2 f\left(\left|\mathbf{x}-\mathbf{x}_{o}\right|\right)+2 w\left(\left|t-t_{o}\right|\right) .
$$

We deduce that $u-\psi$ has a local strict maximum in $\left(\mathbf{x}_{o}, t_{o}\right)$. If $\mathbf{x} \neq \mathbf{x}_{o}$,

$$
D \psi(\mathbf{x}, t)=2 f^{\prime}\left(\left|\mathbf{x}-\mathbf{x}_{o}\right|\right) \frac{\mathbf{x}-\mathbf{x}_{o}}{\left|\mathbf{x}-\mathbf{x}_{o}\right|}
$$

and

$D^{2} \psi(\mathbf{x}, t)=2 f^{\prime}\left(\left|\mathbf{x}-\mathbf{x}_{o}\right|\right) \frac{I}{\left|\mathbf{x}-\mathbf{x}_{o}\right|}+2 f^{\prime \prime}\left(\left|\mathbf{x}-\mathbf{x}_{o}\right|\right) \frac{\left(\mathbf{x}-\mathbf{x}_{o}\right) \otimes\left(\mathbf{x}-\mathbf{x}_{o}\right)}{\left|\mathbf{x}-\mathbf{x}_{o}\right|^{2}}-2 f^{\prime}\left(\left|\mathbf{x}-\mathbf{x}_{o}\right|\right) \frac{\left(\mathbf{x}-\mathbf{x}_{o}\right) \otimes\left(\mathbf{x}-\mathbf{x}_{o}\right)}{\left|\mathbf{x}-\mathbf{x}_{o}\right|^{3}}$.

- If $\mathbf{x}_{h} \neq \mathbf{x}_{o}$, then $D \psi\left(\mathbf{x}_{h}, t\right) \neq 0$, so we have the preceding result

$$
\psi_{t}\left(\mathbf{x}_{h}, n_{h} h\right)-\left|D \psi\left(\mathbf{x}_{h}, n_{h} h\right)\right| G\left(\operatorname{curv}\left(\psi\left(\mathbf{x}_{h}, n_{h} h\right)\right)\right)+o(h) \leq 0,
$$

therefore,

$$
\phi_{t}\left(\mathbf{x}_{o}, t_{o}\right)+2 w^{\prime}\left(\left|n_{h} h-t_{o}\right|\right)-2 f^{\prime}\left(\left|\mathbf{x}_{h}-\mathbf{x}_{o}\right|\right) G\left(\frac{1}{\left|\mathbf{x}_{h}-\mathbf{x}_{o}\right|}\right)+o(h) \leq 0 .
$$

We let $h$ tend to 0 , by definition of $\mathcal{F}(G)$, it remains

$$
\phi_{t}\left(\mathbf{x}_{o}, t_{o}\right) \leq 0 .
$$

- If $\mathbf{x}_{h}=\mathbf{x}_{o}$ for any $h$, we apply 7 with the function $\psi$ defined in step 4 , so we get

$$
g\left(n_{h} h\right)-g\left(\left(n_{h}-1\right) h\right)+f\left(\mathbf{x}_{h}\right)-2 T_{h} f\left(\left|.-\mathbf{x}_{o}\right|\right)\left(\mathbf{x}_{h}\right)+o(h) \leq 0 .
$$

We replace $\mathbf{x}_{h}$ by $\mathbf{x}_{o}$

$$
h g^{\prime}(c)-2 T_{h} f\left(\left|.-\mathbf{x}_{o}\right|\right)\left(\mathbf{x}_{o}\right)+o(h) \leq 0
$$

with $c \in\left[\left(n_{h}-1\right) h ; n_{h} h\right]$. As we assume

$$
\lim _{h \rightarrow 0} \frac{T_{h} f\left(\left|\cdot-\mathbf{x}_{o}\right|\right)\left(\mathbf{x}_{o}\right)}{h}=0
$$

hence

$$
\phi_{t}\left(\mathbf{x}_{o}, t_{o}\right) \leq 0
$$

\section{REFERENCES}

[1] L. Alvarez, F. Guichard, P.-L. Lions and J.-M. Morel, Axioms and fundamental equations of image processing. Arch. Rational Mech. 123 (1993) 199-257.

[2] G. Barles and P.M. Souganidis, Convergence of approximation schemes for fully nonlinear second order equations. Asymptotic Analysis 4 (1991) 271-283.

[3] F. Cao, Partial Differential Equations and Mathematical Morphology. J. Math. Pures Appl. 77 (1998) 909-941.

[4] F. Catte, F. Dibos and G. Koepfler, A Morphological Scheme for Mean Curvature Motion. SIAM J. Numer. Anal. (1995) SINUM 32.6.

[5] Y.-G. Chen, Y. Giga and S. Goto, Uniqueness and existence of viscosity solutions of generalized mean curvature flow equations. J. Differential Geom. 33 (1991) 749-786 
[6] M. Crandall, H. Ishii and P.-L. Lions, User's guide to viscosity solution of second order partial differential equations. Bull. Amer. Math. Soc. (N.S.) 27 (1992) 1-67.

[7] L.C. Evans and J. Spruck, Motion of level sets by mean curvature I. J. Differential Geom. 33 (1991) 635-681.

[8] F. Guichard and J.M. Morel, Partial Differential Equation and image iterative filtering. Tutorial of ICIP 95, Washington D.C. (1995).

[9] H. Ishii and P. Souganidis, Generalized Motion of noncompact hypersurfaces with velocity having arbitrary growth on the curvature tensor. Tohoku Math. J. 47 (1995) 227-250.

[10] J.J. Koenderink, The structure of images. Biol. Cybern. 50 (1984) 363-370.

[11] B.B. Kimia, A. Tannenbaum and S.W. Zucker, Shapes, shocks and deformations. Internat. J. Comput. Vision (1994).

[12] G. Matheron, Random sets and Integral Geometry (John Wiley N.Y., 1975).

[13] Pasquignon D., Computation of skeleton by PDE. IEEE-ICIP, Washington D.C. (1995).

[14] J. Serra, Image Analysis and Mathematical Morphology. Vol. 2, Theoretical Advances, Serra Ed. (London Academic Press, 1988).

[15] A.P. Witkin, Scale space filtering, in Proc. of IJCAI, Karlsruhe (1983) 1019-1021. 